\title{
A Study on Diesel Plug-in Hybrid Vehicle Powertrain Analysis and Modeling
}

\author{
Ho-Un Jeong, Kyu-Hyun Sim, Kwan-Soo Han, and Sung-Ho Hwang
}

\begin{abstract}
TMED (transmission mounted electric device) PHEV(Plug-in hybrid vehicle) powertrain consist of engine, engine clutch, motor, transmission ,differential gear, wheel and tire. By disengaging engine clutch, the vehicle can use only motor power for driving. After battery depleted, engine and motor are cooperate to increase engine efficiency. In this paper, diesel PHEV(Plug-in hybrid vehicle) powertrain dynamic equations was derived from longitudinal vehicle model. In order to design optimal powertrain capacity in given specifications, various capacities of engine and motor model was derived from commercial engine and motor test data. Engine and Motor model use input signal and input shaft speed to calculate torque outputs, also calculate energy conversion efficiency. Using MATLAB Simulink program, diesel PHEV dynamic model was created from engine and motor model, and powertrain dynamic equations. Using developed simulator, we analysis basic performance of diesel PHEV.
\end{abstract}

Index Terms-Diesel plug-in hybrid, vehicle dynamics, vehicle performance analysis.

\section{INTRODUCTION}

PHEV vehicle powertrain uses two types of power source, engine and motor. ICE (Internal combustion engine) convert chemical fuel energy to kinetic energy. Because of engine cycle characteristic and friction loss, conversion efficiency is lower than motor. But fossil fuel has high energy density and easy to store. Otherwise motor convert electric energy to produce kinetic energy with high conversion efficiency, but energy storage system, also called battery, has low energy density. Motor also called generator, and it can convert kinetic energy to electric energy. In vehicle kinetic energy restored into battery by generator, and it called regenerative braking. Optimizing size of engine and motor has to be the first step in vehicle design, because it determines vehicle performance and fuel efficiency [1]-[3].

There was several researches about improve fuel economy of diesel PHEV. First, optimization of powertrain engine and motor size using backward simulation and dynamic programming was adopted in series hybrid bus design. In order to minimize fuel consumption in fixed driving course, feasible region of engine and motor size was selected by using basic dynamics of powertrain. Then make some possible candidate group of engine and motor size to analyze

Manuscript received November 30, 2014; revised May 5, 2015.

Ho-Un Jeong, Kyu-Hyun Sim, and Sung-Ho Hwang are with the School of Mechanical Engineering, Sungkyunkwan University, Suwon, 440-746, Republic of Korea (e-mail: jhoun89@naver.com, dodo@gmail.com, hsh@me.skku.ac.kr).

Kwan-Soo Han is with the Research \& Business Foundation and College of Engineering, Sungkyunkwan University, Suwon, 440-746, Republic of Korea (e-mail:kshansk@skku.edu). fuel economy potential by dynamic programming. Required vehicle performances, acceleration and hill climbing, were verify by forward simulation [4]-[7].

In this paper, the dynamic equations for forward and backward simulation were derived and apply different power size of engine motor model in developed simulator.

\section{DiESEL Plug-IN HYBRID POWERTRAIN}

\section{A. TMED PHEV Structure}

TMED structure is subject of this research. TMED structure consists of engine, engine clutch, motor, battery, transmission and differential gear. Fig. 1 shows TMED PHEV component and arrangement.

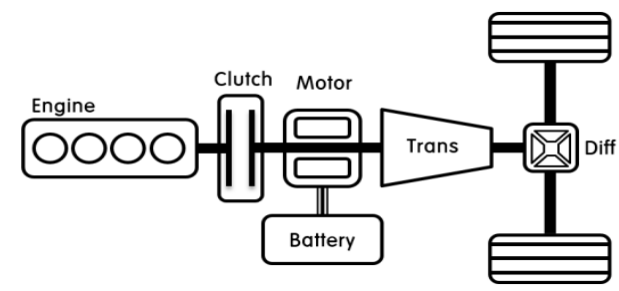

Fig. 1. TMED PHEV structure.

\section{B. Vehicle Parameters}

Subject of research TMED PHEV vehicle parameters are listed in table. Vehicle weight considered addition of motor and battery. The parameter used in calculation of vehicle dynamics equations.

TABLE I: VEHICLE PARAMETERS

\begin{tabular}{lll}
\hline \hline parameters & Value & Unit \\
\hline Vehicle Mass & 1400 & $\mathrm{~kg}$ \\
Coefficient of Drag & 3.3 & \\
Vehicle Projection Area & 2.6 & $\mathrm{~m}^{2}$ \\
Tire Radius & 0.33 & $\mathrm{~m}$ \\
Tire rolling coefficient & 0.01 & \\
\hline \hline
\end{tabular}

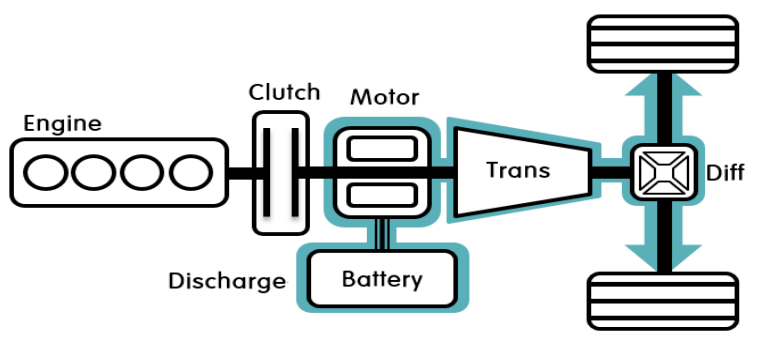

Fig. 2. EV mode energy flow.

\section{Driving Mode}

Because of engine clutch, TMED PHEV can separate 
engine from powertrain. After battery fully charged, vehicle drives in EV mode. Motor use electric energy in battery and EV mode energy flow shows in Fig. 2.

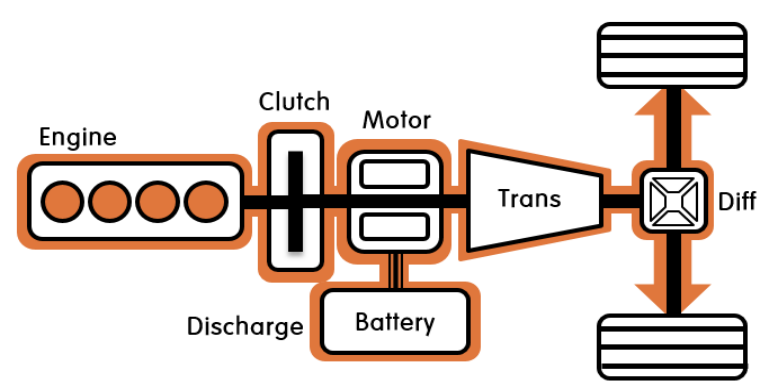

Fig. 3. HEV mode energy flow.

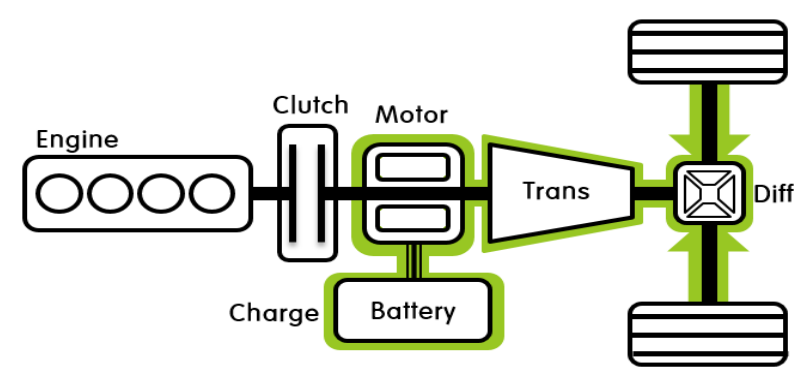

Fig. 4. Regenerative braking mode energy flow.

When SOC decreased under determined limit or driver requires more power than motor creates, HCU (Hybrid control unit) changes to HEV mode. Fig. 3 shows HEV mode power flows. In HEV mode engine starts on and engine clutch is engaged. HCU controls the power distribution ratio. Defend on distribution ratio engine and motor operation points are changed. Changing operation point can improve fuel efficiency. So it is important to control distribution ratio in HEV mode.

When vehicle decelerate, kinematic energy of vehicle restore by regenerative braking. Motor generate reverse

torque to shaft and recharge batteries. Due to difficulty of motor control and battery current capacity.

\section{POWERTRAIN MODELING}

\section{A. Powertrain Modeling}

Longitudinal model of TMED PHEV was made to simulate vehicle's longitudinal behavior. Fig. 5 shows structure and variables of powertrain. Each component has inertia, efficiency and exchange efforts and flows with other component. Engine and motor generates torques. Transmission and differential gears transfer torque to the wheels. In the figure, ' $T$ ' means torque, ' $F$ ' means force, ' $\omega$ ' means rotational speed, ' $v$ ' means vehicle velocity, ' $\eta$ ' means power transfer efficiency, ' $J$ ' means moment of inertia of component and ' $N$ ' means gear ratio. Torque and flow relations between each component were derived in following equations to apply Simulink modeling.

$$
\begin{gathered}
T_{e}-T_{c i}=J_{e} \dot{\omega}_{e} \\
\eta_{c} T_{c i}-T_{c o}=J_{c} \dot{\omega}_{c} \\
T_{c o}+T_{m}-T_{t}=J_{m} \dot{\omega}_{m} \\
\eta_{t} N_{t} T_{t}-T_{d}=J_{t} \dot{\omega}_{t} \\
\eta_{d} N_{d} T_{d}-4 T_{w}=J_{c} \dot{\omega}_{c} \\
4 T_{w}-F_{x} R_{t}=4 J_{w} \dot{\omega}_{w} \\
F_{x}-F_{\text {load }}=m \dot{v}
\end{gathered}
$$

$$
\begin{gathered}
\dot{\omega}_{w}=\frac{1}{R_{t}} \dot{v} \\
\dot{\omega}_{d}=\frac{N_{d}}{R_{t}} \dot{v} \\
\dot{\omega}_{e}=\dot{\omega}_{c}=\dot{\omega}_{m}=\dot{\omega}_{t}=\frac{N_{t} N_{d}}{R_{t}} \dot{v}
\end{gathered}
$$

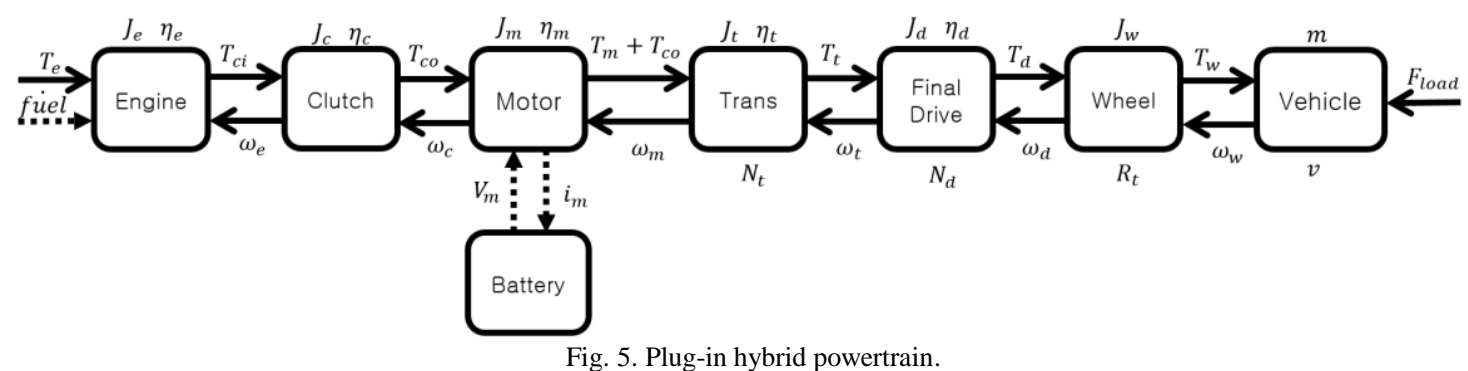

\section{B. Engine Modeling}

Engine model uses two map data. One is output torque depended on engine speed and throttle signal, other is fuel consumption rate depended on output torque and engine speed. Fig. 6 shows engine torque graph. In simulation engine torque response time characteristic was applied by transfer function using engine response time constant.

Engine speed has to be higher than 1000rpm for operation. When engine speed decreased under 500rpm, engine stops. Engine characteristic graphs were extracted from simulation software Autonomie, and scaled by various engine power. Characteristic data were derived by experiment. Using under equation, engine power graph was drawn in Fig. 7. Maximum torque appeared about 2000rpm, and maximum power appeared about $3700 \mathrm{rpm}$.

$$
P_{e}=T_{e} \omega_{e}
$$

Fig. 8 shows engine consumption fuel rate. Fuel rate has $\mathrm{g} / \mathrm{s}$ unit. In the simulation fuel rate will be integrated to calculate fuel consumption amount. When calculated fuel consumption exceed initial fuel weight, vehicle could not proceeds and simulation will be stopped.

Engine output torque and fuel rate is essential for engine modeling. But, in order to calculate engine efficiency BSFC (Brake Specific Fuel Consumption) was calculated. BSFC means rate of fuel consumption divided by engine output 
power. Engine BSFC and Efficiency were calculated by under equations, where $\gamma$ is fuel rate.

$$
\begin{gathered}
B S F C=\frac{\gamma}{T_{e} \omega_{e}} \\
\eta_{e}=\frac{1}{B S F C \cdot 0.0119531}
\end{gathered}
$$

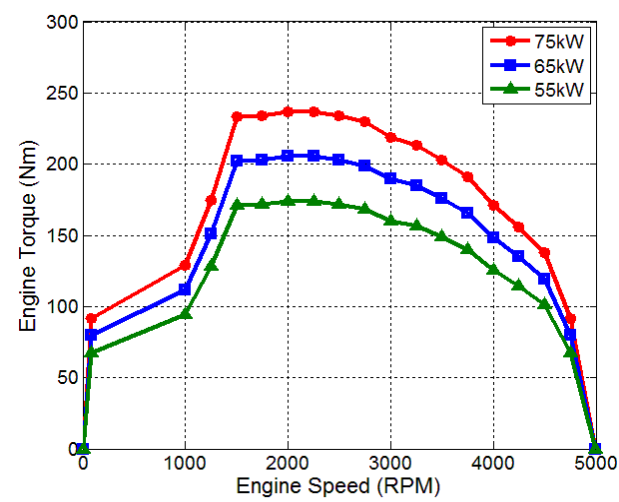

Fig. 6. Engine torque-speed.

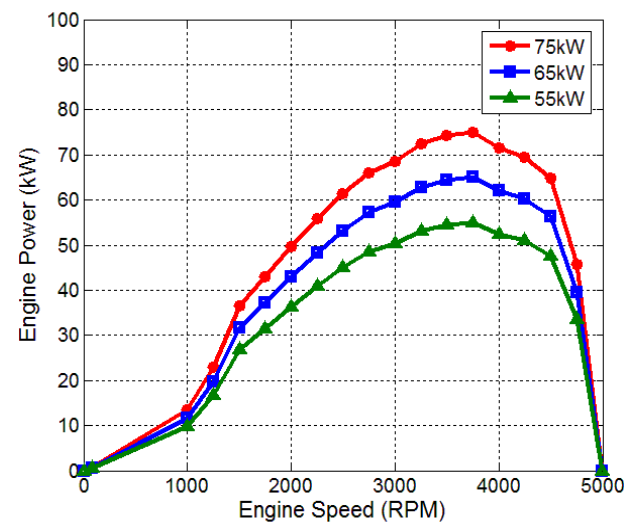

Fig. 7. Engine power-speed.

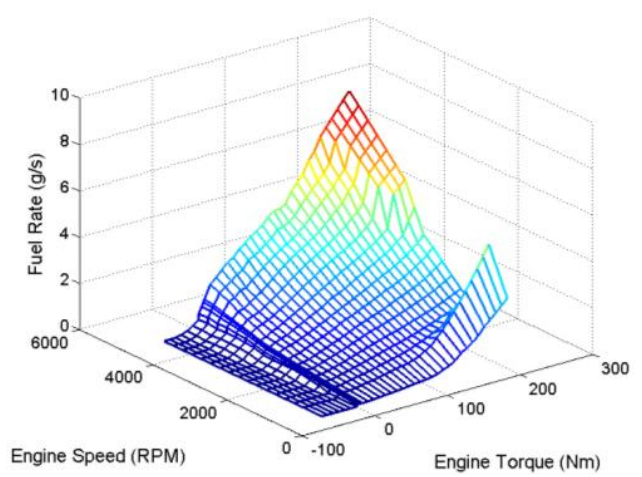

Fig. 8. $65 \mathrm{~kW}$ engine fuel rate.

\section{Motor Modeling}

Motor model was made in similar stage with engine model. Motor torque depended on motor speed and demanded torque signal. Fig. 11 shows motor peak output torque and Fig. 12 shows motor peak output power. As we can see, power output increased proportionally fewer than $2000 \mathrm{rpm}$, and it is called constant torque region [8]. When motor speed exceed over $2000 \mathrm{rpm}$ output power maintained, called constant power region. In simulation motor acting like generator, when regenerative braking, engine makes reverse torque. Generator makes electricity and torque response time characteristic was applied by transfer function using motor response time constant.

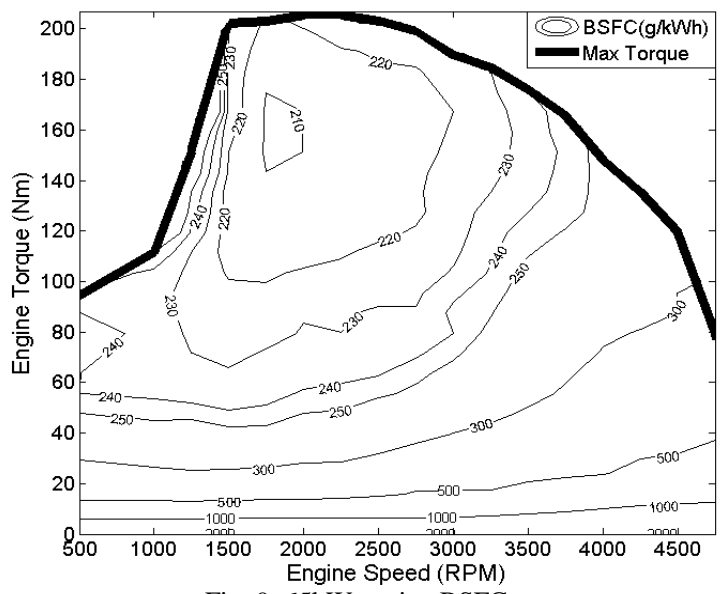

Fig. 9. $65 \mathrm{~kW}$ engine BSFC.

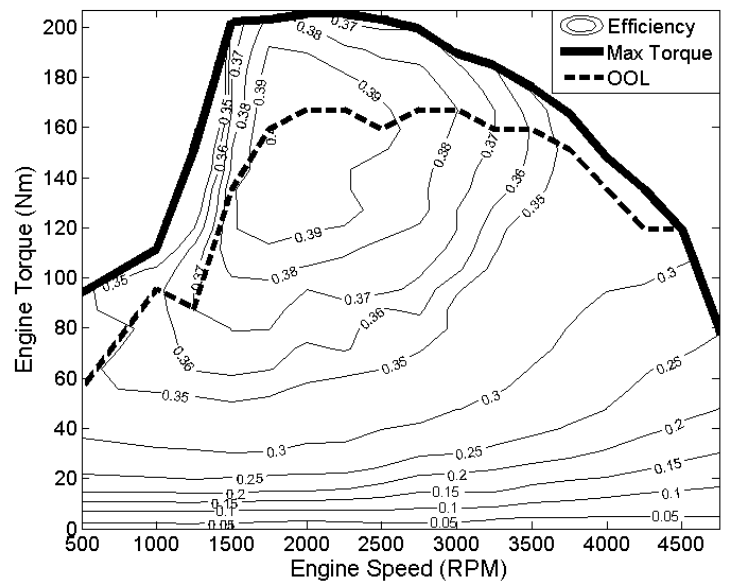

Fig. 10. $65 \mathrm{~kW}$ engine efficiency and optimal operation line.

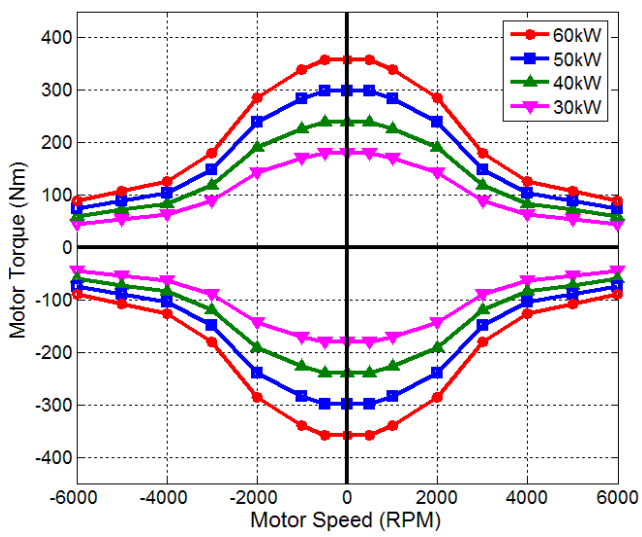

Fig. 11. Motor peak torque-speed.

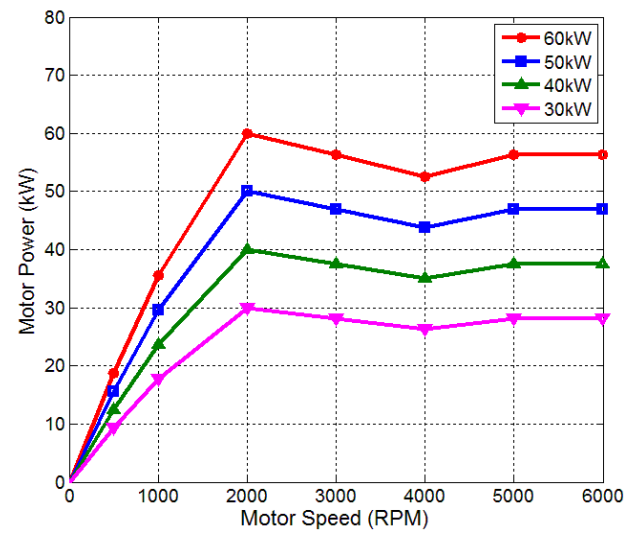

Fig. 12. Motor peak power-speed. 


\section{DeVElopment OF SimulinK MOdEL}

Using Simulink program, TMED PHEV longitudinal model was developed. Simulator structure composed of each component model. $4 \mathrm{kWh}$ Battery model included for the EV mode range limitation. Engine and motor model creates torque, and clutch, transmission, differential gear model transfer torque and shaft speed calculated by derived equations. In vehicle model summation of longitudinal force makes acceleration of vehicle. By integrating acceleration, vehicle speed was calculated and. In Driver model consist of acceleration and brake signal creator, engine and motor control unit, hybrid mode control unit [9], [10].

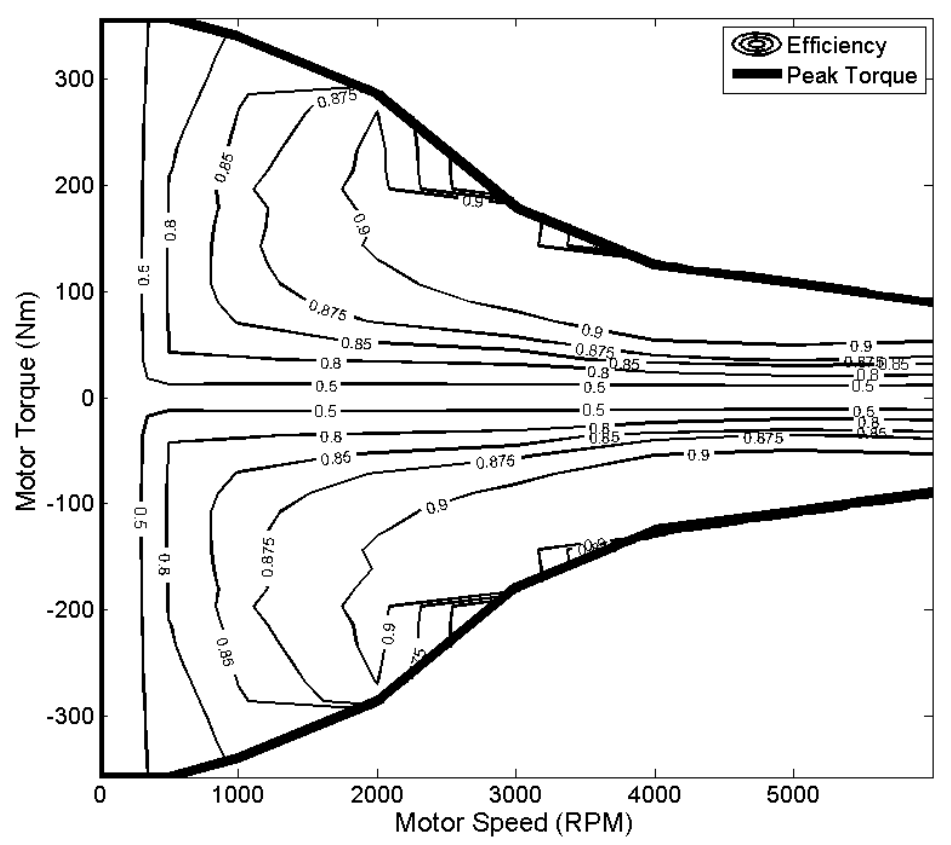

Fig. 14. 60kW motor efficiency.

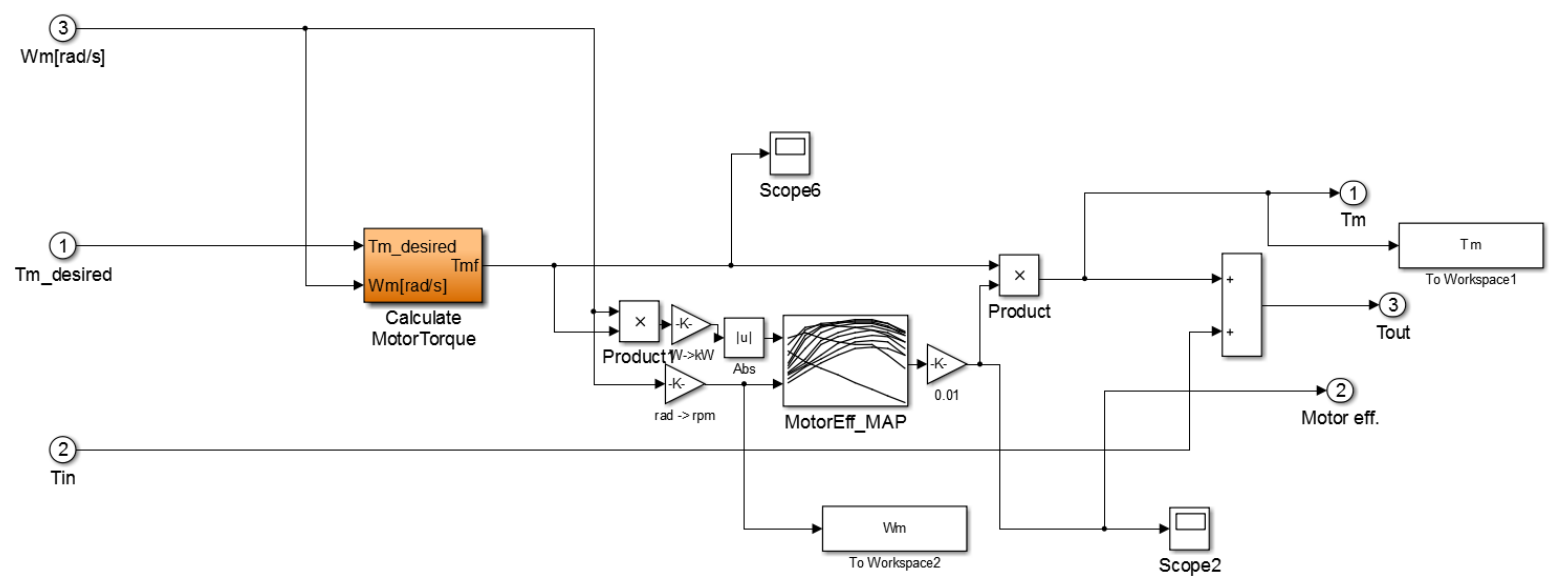

Fig. 13. Simulink motor model.

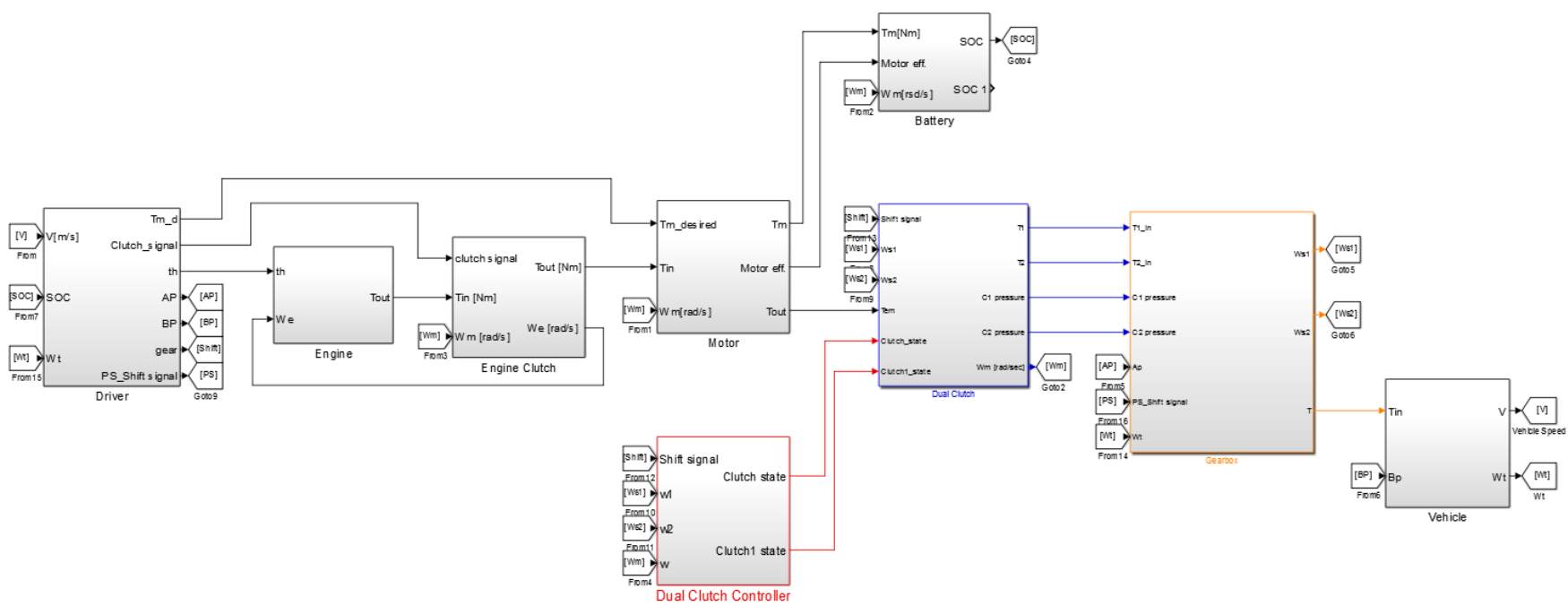

Fig. 14. Simulink TMED PHEV model. 


\section{CONCLUSION}

In this paper we analyze TMED PHEV structure and driving mode. Studied object can have EV mode by using engine clutch. To evaluate powertrain longitudinal dynamic behavior and fuel economy, powertrain dynamic equation was derived, and using that equation, simulator model was developed. Engine and Motor model uses torque, fuel, power map data extracted from experiment data and scaled by maximum power. Simulink model will be use to compare various type of engine and motor size combination about acceleration performance, hill climbing ability and fuel economy. By compare these parameters, we anticipate to find optimal power capacitance of given hybrid type.

\section{REFERENCES}

[1] J. Park et al., "Development of parallel type diesel-based mild hybrid electric vehicle," in Proc. KSAE Autumn Conference, 2005, pp. 1408-1412.

[2] S. Park et al., "Experiment investigation for fuel efficiency and performance of diesel hybrid electric vehicle," in Proc. KSAE Autumn Conference, 2006, pp. 1603-1610.

[3] K. Yeom et al., "Optimization of the parallel diesel hybrid vehicle," Transactions of KSAE, vol. 16, no. 6, pp. 26-32, 2008.

[4] J. Yang et al., "Simulation for the fuel economy of parallel diesel hybrid vehicle," in Proc. KSAE Spring Conference, 2007, pp. 370-375.

[5] M. Kim et al., "Fuel economy up strategy and effect on 1.7L class hybrid diesel engine," in Proc. KSAE Autumn Conference, 2013, pp. 131-132.

[6] T. Bae et al., "PHEV power train design and fuzzy logic control for optimal engine drive," in Proc. KORMARC Conference, 2011, pp. 489-490.

[7] N. Kim et al, "A backward simulator for calculating optimal control trajectories," in Proc. KSAE Autumn Conference, 2009, pp. 1498-1503.

[8] S.-S. Kim et al., "Development of fchev virtual platform using motor model based on mathematical formulation," Transactions of KSAE, vol. 21, no. 6, pp. 31-39, 2013.

[9] J. J. Baek et al., "Development of performance simulator for 6-speed DCT-based hybrid electric vehicle to evaluate the fuel economy," $J$. Korean Soc. Fluid Power Constr. Equip., vol. 10, no. 4, pp. 1-6, 2013.
[10] J. J. Baek et al., "Development of performance simulator for 6-speed dct-based hybrid electric vehicle," in Proc. KSFC Autumn Conference, pp. 41-45, 2013.

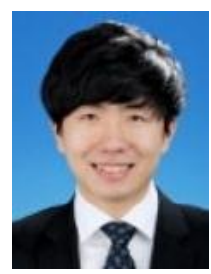

Ho-Un Jeong received the B.S. degree in mechanical engineering from Sungkyunkwan University, Suwon, Korea, in 2013. He is currently working toward the M.S. degree with the School of Mechanical Engineering, Sungkyunkwan University, Suwon, Korea. His research interests include the plug-in hybrid vehicle powertrain, dual clutch transmission and optimization.

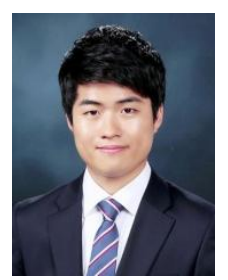

Kyu-Hyun Sim received the B.S. degree in mechanical engineering from Sungkyunkwan University, Suwon, Korea, in 2013. He is currently working toward the M.S. degree with the School of Mechanical Engineering, Sungkyunkwan University, Suwon, Korea. His research interests include the hybrid vehicle powertrain and optimization.

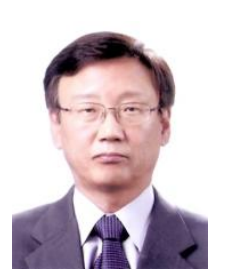

Kwan-Soo Han received the B.S. degree in mechanical engineering from Seoul National University, Seoul, Korea, in 1977, the diploma and Dr.-ing degree from Technical University Berlin, Berlin, Germany in 1991 , 1999 respectively. He is a professor at Research \& Business Foundation and at College of Engineering, Sungkyunkwan University, Suwon, Korea.

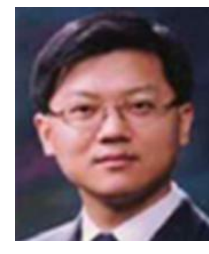

Sung-Ho Hwang received the B.S. degree in mechanical design and production engineering, the M.S. and Ph.D. degrees in mechanical engineering from Seoul National University, Seoul, Korea, in 1988 , 1990 , and 1997, respectively. He is currently an associate professor with the School of Mechanical Engineering, Sungkyunkwan University, Suwon, Korea. 